\title{
The plasma biomarker soluble SIGLEC-1 is associated with the type I interferon transcriptional signature, ethnic background and renal disease in systemic lupus erythematosus
}

João J. Oliveira ${ }^{1+}$, Sarah Karrar ${ }^{2 \dagger}$, Daniel B. Rainbow ${ }^{1,3}$, Christopher L. Pinder ${ }^{2}$, Pamela Clarke ${ }^{1}$, Arcadio Rubio García 1,3, Osama Al-Assar ${ }^{3}$, Keith Burling ${ }^{4}$, Sian Morris ${ }^{5}$, Richard Stratton ${ }^{5}$, Tim J. Vyse ${ }^{2}$, Linda S. Wicker ${ }^{1,3}$, John A. Todd ${ }^{1,3}$ and Ricardo C. Ferreira ${ }^{1,3^{*}}$ (D)

\begin{abstract}
Background: The molecular heterogeneity of autoimmune and inflammatory diseases has been one of the main obstacles to the development of safe and specific therapeutic options. Here, we evaluated the diagnostic and clinical value of a robust, inexpensive, immunoassay detecting the circulating soluble form of the monocyte-specific surface receptor sialic acid binding Ig-like lectin 1 (sSIGLEC-1).

Methods: We developed an immunoassay to measure sSIGLEC-1 in small volumes of plasma/serum from systemic lupus erythematosus (SLE) patients $(n=75)$ and healthy donors $(n=504)$. Samples from systemic sclerosis patients $(n=99)$ were studied as an autoimmune control. We investigated the correlation between SSIGLEC- 1 and both monocyte surface SIGLEC-1 and type I interferon-regulated gene (IRG) expression. Associations of sSIGLEC-1 with clinical features were evaluated in an independent cohort of SLE patients $(n=656)$.

Results: Plasma concentrations of SSIGLEC-1 strongly correlated with expression of SIGLEC-1 on the surface of blood monocytes and with IRG expression in SLE patients. We found ancestry-related differences in SSIGLEC-1 concentrations in SLE patients, with patients of non-European ancestry showing higher levels compared to patients of European ancestry. Higher sSIGLEC-1 concentrations were associated with lower serum complement component 3 and increased frequency of renal complications in European patients, but not with the SLE Disease Activity Index clinical score.
\end{abstract}

Conclusions: Our SSIGLEC-1 immunoassay provides a specific and easily assayed marker for monocyte-macrophage activation, and interferonopathy in SLE and other diseases. Further studies can extend its clinical associations and its potential use to stratify patients and as a secondary endpoint in clinical trials.

Keywords: Soluble SIGLEC-1, Biomarker, Autoimmunity, Type I interferon, Interferonopathy

\footnotetext{
* Correspondence: ricardo.ferreira@well.ox.ac.uk

${ }^{\dagger}$ João J. Oliveira and Sarah Karrar contributed equally to this work. 'Department of Medical Genetics, JDRF/Wellcome Diabetes and Inflammation Laboratory, NIHR Cambridge Biomedical Research Centre, Cambridge Institute for Medical Research, University of Cambridge, Cambridge, UK

${ }_{3}^{3}$ JDRF/Wellcome Diabetes and Inflammation Laboratory, Wellcome Centre for Human Genetics, Nuffield Department of Medicine, NIHR Oxford Biomedical Research Centre, University of Oxford, Roosevelt Drive, Oxford, UK Full list of author information is available at the end of the article
}

(c) The Author(s). 2018 Open Access This article is distributed under the terms of the Creative Commons Attribution 4.0 International License (http://creativecommons.org/licenses/by/4.0/), which permits unrestricted use, distribution, and reproduction in any medium, provided you give appropriate credit to the original author(s) and the source, provide a link to the Creative Commons license, and indicate if changes were made. The Creative Commons Public Domain Dedication waiver (http://creativecommons.org/publicdomain/zero/1.0/) applies to the data made available in this article, unless otherwise stated. 


\section{Background}

The type I interferon (IFN) pathway was identified as a central feature of the autoimmune disease systemic lupus erythematosus (SLE) when IFN- $\alpha$ was first detected at high levels in patients' sera [1]. Since this initial observation, the development of SLE-like clinical manifestations in patients treated with IFN- $\alpha$ for different malignancies pointed to the involvement of IFN- $\alpha$ in the aetiology of the disease [2]. Furthermore, naturally occurring anti-IFN- $\alpha$ antibodies in SLE patients have been shown to be associated with milder forms of the disease [3]. The identification of a constitutive IFN transcriptional signature comprising hundreds of IFN-regulated genes (IRGs) in peripheral blood from a subset of SLE patients [4, 5] suggested that the IFN signature could be used as a clinical biomarker to stratify patients with autoimmune and inflammatory diseases in which type I IFNs are known to play a pathogenic role, referred to as the interferonopathies. Nevertheless, the precise link between the IFN signature and molecular subtypes of disease or with broader disease severity scores has been put into question [6]. With the development of more sophisticated high-throughput genomic tools, it has become apparent that the IFN signature is a complex composite marker, which can be further stratified into several distinct signatures that are better predictors of disease subtype [7]. Longitudinal analyses have revealed that the IFN signature is highly variable over time as a result of alterations in blood composition caused by therapy or progression of the disease $[7,8]$. This is particularly relevant to IFN-driven diseases, as IFN- $\alpha$ is known to significantly alter the relative distribution of immune cell types in blood, which can severely compromise the diagnostic potential of the whole blood IFN signature [8] and lead to the observed lack of correlation between the signature and disease activity over time $[9,10]$.

These findings have led to the investigation of other potential cell-type specific biomarkers that could be better predictors of disease severity or clinical subtypes. One such IFN-regulated marker that has shown promise for the stratification of SLE patients is sialic acid binding Ig-like lectin 1 (SIGLEC-1) [11-13]. SIGLEC-1 is a cell-adhesion molecule involved in the initial contacts with sialylated pathogens and mediates phagocytosis and endocytosis of pathogens, thereby promoting efficient immune responses to limit infection [14]. Unlike the canonical IFN transcriptional signature, which is a composite of many genes expressed at different levels in different immune cells, SIGLEC-1 is expressed exclusively in cells of the myeloid lineage, namely tissue-resident macrophages and monocyte-derived dendritic cells $[15,16]$. In blood, expression of surface SIGLEC- 1 is restricted to CD14 ${ }^{+}$ monocytes, and has been previously reported to be increased in several other autoimmune diseases, including rheumatoid arthritis [17], systemic sclerosis (SSc) [18] and primary biliary cirrhosis [19]. In addition, a recent study has shown that increased SIGLEC-1 expression on the surface of monocytes was a predictor of congenital heart block during pregnancy in children from Ro/SS-A autoantibody-positive mothers [20].

These data support the use of SIGLEC-1 as a potential cell-type specific biomarker for the stratification of patients with an overt type I IFN response. However, assay of surface SIGLEC-1 requires intact cells and flow cytometry, features that are not conducive for the development of a high-throughput, inexpensive biomarker assay, ideally detectable in plasma/serum. Here we show that a circulating form of SIGLEC-1 can be detected in serum/plasma, and develop a robust and inexpensive immunoassay to measure its concentration. Furthermore, we provide evidence that the concentration of soluble SIGLEC-1 (sSIGLEC-1) is associated with the patient's ancestry and with renal involvement in SLE patients. Therefore, sSIGLEC-1 is a new circulating plasma/serum biomarker of type I IFN activity in systemic autoimmune, inflammatory and infectious diseases that can be used accurately and conveniently in large numbers of samples, and could be used in clinical trials of drugs modulating the IFN signalling pathway for patient stratification and as a secondary endpoint.

\section{Methods \\ Participants}

Discovery cohort (cohort 1) study participants included 34 SLE patients (median age 39 years, range 20-72 years; 32/34 female) recruited from Guy's and St Thomas' NHS Foundation Trust. All patients satisfied ACR SLE classification criteria and were allocated a disease activity using SLEDAI-2K at the time of sampling. Patients were recruited from a clinic in which the severity of disease was such that none of the patients was on high-dose oral corticosteroids (>15 mg/day) or B-cell depleting therapy. Healthy volunteers matched for age and sex $(n=$ 24 ; median age 43 years, range $25-60$ years; $23 / 24$ female) were recruited from the Cambridge BioResource.

A replication cohort (cohort 2) of 41 SLE patients (median age 52 years, range $21-82$ years; 38/41 female) and 490 healthy volunteers (median age 48 years, range 18-78 years; $320 / 490$ female) was recruited from the Cambridge BioResource. The SLE patients were recruited specifically for this study outside their regular clinic visits, and were otherwise well at the time of bleeding. No additional disease information or ancestry data were available for this cohort of patients.

To investigate the association between sSIGLEC-1 with ancestry and clinical manifestations, a third independent cohort (cohort 3$)$ of SLE patients $(n=656$; median age 45 years, range $15-82$ years; 592/655 female, one unknown) 
was recruited from multiple collaborative centres in the UK (St Thomas's Hospital, Newcastle Hospital, City Hospital Sunderland, City Hospital Birmingham, Royal Hallamshire Hospital, Hammersmith Hospital, West Middlesex Hospital and Basildon Hospital). Most patients were of European $(n=370)$, South East Asian $(n=134)$, African/Afro-Caribbean $(n=94)$ and Far East Asian $(n=$ 19) ancestries. Twenty-three patients were of other minor ancestry groups (including Middle Eastern, Maori and Fiji ancestry) and 16 had missing ancestry information. The history of patients' clinical manifestations since their disease diagnosis up to the time of their visit is summarised in Table 1.

A cohort of systemic sclerosis (SSc) patients $(N=99)$, stratified into patients with limited cutaneous SSc $(N=50)$ or diffuse SSc $(N=49)$, and a matching cohort of healthy donors $(N=50)$ were recruited from the Royal Free Hospital, UCL, London. All patients had a definite diagnosis of SSc according to the 2013 ACR/EULAR SSc classification criteria [21].

All samples and information were collected with written and signed informed consent after approval from the relevant research ethics committees (REC numbers 05/ Q0106/20, 07/H0718/49 and 08/H0308/153; and NHS Health Research Authority, NRES Committee LondonHampstead, HRA, REC number 6398, Investigating the pathogenesis of systemic sclerosis).

\section{Flow cytometry}

SIGLEC-1 expression was measured in peripheral blood mononuclear cells (PBMCs) from 34 SLE patients and 24 healthy donors from the discovery cohort. PBMCs were thawed in a $37{ }^{\circ} \mathrm{C}$ water bath, resuspended in X-VIVO 15 (Lonza) + 1\% heat-inactivated, filtered, human $A B$ serum (Sigma) and immunostained for $30 \mathrm{~min}$ at room temperature. SIGLEC-1 expression on CD14 ${ }^{+}$ monocytes was determined using fluorochromeconjugated antibodies against CD14 (Clone M5E2; BioLegend) and SIGLEC-1 (Clone 7-239; BioLegend). Immunostained samples were acquired using a BD LSR Fortessa (BD Biosciences) flow cytometer, and data were analysed using FlowJo (Tree Star). Dead cells were excluded based on the eFluor780 Fixable Viability Dye (eBiosciences).

\section{Soluble SIGLEC-1 time-resolved fluorescence immunoassay}

Plasma/serum sSIGLEC-1 concentrations were measured using a non-isotopic time-resolved fluorescence (TRF) assay based on the dissociation-enhanced lanthanide fluorescent immunoassay technology (DELFIA; PerkinElmer). Duplicate test plasma/serum samples diluted 1:10 in assay buffer (PBS, 0.05\% Tween-20, 10\% FCS) were incubated for $2 \mathrm{~h}$ at room temperature and then at $4{ }^{\circ} \mathrm{C}$ overnight in 96-well EIA/RIA plates (Corning) coated with $1 \mu \mathrm{g} / \mathrm{ml}$ mouse monoclonal anti-human SIGLEC-1 capture antibody (AB18619; Abcam). Sample detection was performed using a biotinylated sheep polyclonal detection antibody (BAF5197; R\&D Systems) diluted to a final concentration of $200 \mathrm{ng} / \mathrm{ml}$. Following incubation with the secondary antibody, europium-labelled streptavidin (PerkinElmer) was added and the concentration of antigen was measured by the amount of disassociated europium that is fluorescent at $615 \mathrm{~nm}$ after excitation at $320 \mathrm{~nm}$.

Quantification of test samples was obtained by fitting the readings to a human recombinant SIGLEC-1 (R\&D Systems) serial dilution standard curve on each plate $\left(r^{2}>0.995\right)$. To maintain assay consistency, the recombinant protein was aliquoted and stored at $-80{ }^{\circ} \mathrm{C}$ immediately following reconstitution and a fresh aliquot from the same lot was used for each assay.

Table 1 Summary of the history of clinical manifestation of the SLE patients in cohort 3

\begin{tabular}{|c|c|c|c|c|c|c|}
\hline \multirow[t]{2}{*}{ Phenotype } & \multicolumn{2}{|l|}{ EUR } & \multicolumn{4}{|c|}{ Non-EUR } \\
\hline & $\bar{N}$ & Affected patients, $n(\%)$ & $\bar{N}$ & Affected patients, $n$ (\%) & $\mathrm{OR}^{\mathrm{a}}(95 \% \mathrm{Cl})$ & $P$ value ${ }^{b}$ \\
\hline Renal & 320 & $75(24.4 \%)$ & 243 & $81(33.3 \%)$ & $1.60(1.11-2.32)$ & 0.01 \\
\hline Neurological & 330 & 36 (10.9\%) & 244 & $42(17.2 \%)$ & $1.67(1.05-2.74)$ & 0.03 \\
\hline Haematological & 366 & $185(50.5 \%)$ & 268 & 106 (39.6\%) & $0.65(0.47-0.89)$ & 0.007 \\
\hline dsDNA positivity & 370 & $143(38.6 \%)$ & 270 & $83(30.7 \%)$ & $0.70(0.51-0.98)$ & 0.04 \\
\hline Admission rate ${ }^{c}$ & 351 & $140(39.9 \%)$ & 196 & $86(43.9)$ & $1.20(0.84-1.71)$ & 0.31 \\
\hline Biologics ever needed ${ }^{d}$ & 332 & $23(6.9 \%)$ & 239 & $12(5.0 \%)$ & $0.70(0.34-1.43)$ & 0.33 \\
\hline
\end{tabular}

Summary of available clinical history for the 656 study participants from cohort 3 since their disease diagnosis and up to the time of their visit. Data were stratified by study cohort

CI confidence interval, EUR patients of European ancestry, non-EUR patients of non-European ancestry, SLE systemic lupus erythematosus

${ }^{a}$ Odds ratio $(\mathrm{OR})$ in non-European patients

${ }^{\mathrm{b}} P$ values calculated using Pearson's chi-squared test

'Admission defined as any patient requiring hospital admission specifically for SLE in the 5 years prior to and including the date of their clinic visit at which the blood sample was taken

${ }^{\mathrm{d}}$ Patients treated with biologic drugs at any time since their disease diagnosis 
The lower limit of detection of the assay was set as $2 \mathrm{x}$ background levels in each plate and corresponded to an average of $1.29 \mathrm{ng} / \mathrm{ml}$ across all plates. Samples with measurements below the limit of detection (6/589) were set to $1.29 \mathrm{ng} / \mathrm{ml}$. Assay specificity was confirmed using a biotinylated sheep polyclonal isotype control (R\&D Systems). Technical variation was assessed in duplicate measurements of all samples (average $\mathrm{CV}=5.0 \%$ ). Samples with CV $>30 \%$ between duplicates (10/589) were excluded from the analysis. To evaluate potential matrix effects, we diluted a sample with high sSIGLEC-1 concentration with assay medium and showed a linear titration between 1:2 and 1:16 dilutions $\left(r^{2}=0.94\right)$.

\section{IFN- $a_{2 b}$ single-molecule digital ELISA assay}

Circulating levels of IFN- $\alpha_{2 b}$ were measured by SingleMolecule Array (SIMOA) digital ELISA (Quanterix) according to the manufacturer's instructions. IFN- $\alpha$ detection was achieved using mouse anti-IFN- $\alpha$ monoclonal antibodies: a neutralising antibody (clone MMHA-capture) and an anti-IFN- $\alpha$ antibody raised against an IFN- $\alpha_{2 b}$ antigen (clone 7 N41-detection). Cross-reactivity to the other IFN- $\alpha$ subtypes was not assessed. Measurements were performed in plasma samples, which had never been previously thawed, from the 41 SLE patients of cohort 2 .

\section{Type I IFN transcriptional signature in PBMCs}

RNA from 34 SLE patients and 24 age and sex-matched healthy donors in cohort 1 and from 41 SLE patients and 41 age and sex-matched healthy donors in cohort 2 was extracted from freshly isolated PBMCs stored in TRIZOL immediately after collection, using the Direct-zol RNA Mini-Prep kit (Zymo Research) following the manufacturer's instructions. The RNA concentration was measured by NanoDrop (Thermo Fisher Scientific), and $50 \mathrm{ng}$ of total RNA were hybridised with a custom NanoString CodeSet (NanoString Technologies), containing 56 IRGs previously identified as discriminative of the IFN signature [22]. Expression levels were assessed using an nCounter Flex instrument (NanoString Technologies). Data were processed using the nSolver Analysis Software following normalisation to the geometric mean of positive control spike-ins and the gene expression of eight housekeeping genes.

Expression of 56 IRGs previously identified as discriminative of the IFN signature [22] were assessed, with a custom NanoString CodeSet (NanoString Technologies), using RNA from 34 SLE patients and 24 age and sex-matched healthy donors in cohort 1 and from 41 SLE patients and 41 age and sex-matched healthy donors in cohort 2 .

A quantitative metric of the IFN signature was generated using principal component analysis by projection of the expression of the 56 IRGs onto the first principal component (PC1), which was found to explain $86.3 \%$ of the variance of this dataset. A complete list of the 56 IRGs and respective NanoString probe sequences is presented in Additional file 1.

\section{Statistical analyses}

Statistical analyses were performed using Prism software (GraphPad) and R software (https://www.r-project.org). Given that most phenotypes showed moderate to strong right skew that violated the assumption of normality, the phenotypes were log-transformed before statistical testing and all reported values refer to the geometric mean of the respective measurements.

\section{Cohorts 1 and 2}

The association of sSIGLEC-1 and measured immune parameters in cohorts 1 and 2 was performed using twotailed non-parametric Mann-Whitney tests. Correlation analyses were performed using linear regression on the log-transformed data.

\section{Cohort 3}

All statistical analyses with the clinical data available for the 656 patients from cohort 3 were performed using $\mathrm{R}$ software. Association between ancestry and sSIGLEC-1 concentration was assessed using a two-tailed Student's $t$ test. The odds ratio (OR) of each clinical parameter (history of admission within 5 years; ever having suffered with renal, haematological or neurological disease; requiring biological therapy or active corticosteroid use to control disease) in European and non-European patients was assessed by Pearson's chi-squared test.

Patients were divided into groups based on sSIGLEC-1 serum level centiles $(<50$ th centile, 51st-74th centile, 75 th -95 th centile and $>95$ th centile). Association of each group and clinical parameters was performed using a logistical regression model. Patients of European and non-European ancestry were analysed separately as ethnicity was a major confounding factor.

Association of the sSIGLEC-1 concentration with other serological parameters of disease (levels of C3 and C4 measured within 3 months of the visit at the patient's local centre/hospital, anti-dsDNA antibody titres and C-reactive protein) and the estimated glomerular filtration rate (eGFR) were assessed using linear regression on the log-transformed data. Association of sSIGLEC-1 concentration and renal disease activity was also determined, based on the clinical notes documentation, at the time of the sample collection and the last documented episode of active nephritis. Comparison of the different disease activity groups was done using two-tailed Student's $t$ tests comparing the mean sSIGLEC-1 concentration of each group to the control group of patients who never developed renal complications. 


\section{Results}

\section{Soluble SIGLEC-1 assay development}

To investigate whether we could detect SIGLEC-1 expression levels in peripheral blood, we developed an immunoassay based on TRF to measure the concentrations of the circulating form of the receptor, which we refer to as sSIGLEC-1. Although SIGLEC1 is predicted to encode a soluble protein isoform, it has not been previously shown that such a soluble protein can be detected in plasma/serum.

We found that sSIGLEC-1 was detected in the circulation, with concentrations ranging from 1.29 to $276.1 \mathrm{ng} / \mathrm{ml}$ in plasma/serum samples from healthy donors and SLE patients. Technical variation of the assay was found to be very low, as assessed by two independent measurements of the same plasma sample from 23 healthy donors performed 308 days apart (median $C V=4.8 \%, r^{2}=0.91$; Fig. 1a), indicating minimal inter-assay variability. Similarly, biological sSIGLEC-1 levels were found to be very stable $(\mathrm{CV}=$ $11.8 \%, r^{2}=0.67$; Fig. 1b) in 19 healthy donors bled at two separate visits (median time between visit 378 days, range 239-519 days), with only two donors showing physiological differences $(\mathrm{CV}>20 \%)$ between visits, likely due to viral infections [22]. Of note, one donor showed high concentrations of sSIGLEC-1 on the first visit $(28.3 \mathrm{ng} / \mathrm{ml})$, which were maintained 343 days after the initial measurement (23.4 ng/ml; Fig. 1b), suggesting that, in addition to viral and possibly non-viral infections, genetic factors regulate the sSIGLEC-1 levels.

To expand the applicability of the sSIGLEC-1 immunoassay, we also developed an electrochemical luminescencebased (ECL) assay on the Meso Scale Discovery (MSD) platform, using the same detection antibodies and experimental protocol. The assay working range was found to be $0.5-1000 \mathrm{ng} / \mathrm{ml}$, with an approximate $92 \%$ recovery of recombinant SIGLEC-1 protein spiked into serum samples. Stability of the assay over time was assessed using three pools of serum samples with increasing sSIGLEC-1 concentrations that were measured over six different assays run over a 2-day period. Assay stability was consistent with the TRF assay, with CVs of $7.7 \%, 5.6 \%$ and $8.5 \%$ for the low, medium and high QC pools, respectively (Fig. 1c). Furthermore, we found a very high concordance between the TRF and ECL assays $\left(r^{2}=0.78\right.$; Fig. $\left.1 d\right)$, as assessed by measuring a subset of 41 SLE patients on both platforms, using two independent serum aliquots.

\section{Soluble SIGLEC-1 levels are correlated with the surface SIGLEC- 1 expression on CD14 ${ }^{+}$monocytes}

Currently, the surface expression of SIGLEC-1 on monocytes has been suggested as a sensitive cell-type specific biomarker for SLE in blood [13]. To investigate the relationship between surface and soluble SIGLEC-1 levels, we immunophenotyped the expression of this protein on the surface of $\mathrm{CD} 14^{+}$monocytes in PBMCs collected from a discovery cohort (cohort 1) of 34 SLE patients and 24 matched healthy donors (Fig. 2a). Consistent with previous findings $[12,13]$, we found that the surface expression of SIGLEC-1 was increased in CD14 ${ }^{+}$ monocytes from SLE patients compared to healthy controls $\left(P=1.4 \times 10^{-4}\right.$; Fig. 2b). However, we found bimodal expression of the surface SIGLEC-1 among SLE patients, with 10 subjects (10/34, 29\%; Fig. 2b) presenting low levels of protein expression, similar to the ranges observed in healthy donors, and the rest presenting much higher levels, rarely observed in healthy volunteers (Fig. 2a, b).

We found a strong correlation between the surface expression of SIGLEC-1 on monocytes and the concentration of sSIGLEC-1 in plasma samples from the same donors, particularly among SLE patients $\left(r^{2}=0.73, P=\right.$ $7.9 \times 10^{-10}$; Fig. $2 \mathrm{c}$ ). The distribution of sSIGLEC-1 levels recapitulated the same bimodal distribution of the surface SIGLEC-1 expression on SLE patients, ranging from 'low normal' physiological levels observed in healthy donors to the very high levels observed in a subset of patients (Fig. 2c).

\section{Association of sSIGLEC-1 with the IFN transcriptional signature and SLE Disease Activity Index}

To assess whether sSIGLEC-1 was associated with the IFN signature, we measured the transcription of 56 IRGs previously found to recapitulate the IFN signature [22]. We found that sSIGLEC-1 levels were significantly correlated with the IFN transcriptional signature in PBMCs from SLE patients $\left(r^{2}=0.67, P=2.9 \times 10^{-9}\right.$; Fig. 3a). The correlation was also observed in healthy donors $\left(r^{2}=0.34\right.$, $P=2.8 \times 10^{-3}$; Fig. 3a), albeit to a lower extent. These findings were replicated in an independent cohort (cohort 2) of 41 SLE patients $\left(r^{2}=0.46, P=1.2 \times 10^{-6}\right.$; Fig. 3b), confirming that sSIGLEC-1 is a marker of the peripheral IFN signature. The lower correlation in the replication cohort is consistent with a lower overall disease severityand concomitant lower sSIGLEC-1 levels-of the patients in the replication cohort, who were recruited outside their regular clinic visits.

Recently, a single-molecule digital ELISA assay has been shown to detect IFN- $\alpha$ at femtomolar levels in the circulation even from healthy individuals [23]. Consistent with its potent biological activity, over a third of the SLE patients showed very low IFN- $\alpha_{2 b}$ concentrations $(<10 \mathrm{fg} / \mathrm{ml}$; Fig. $3 \mathrm{c}$ ), which were close to the reported limit of detection. In our hands, the assay showed good reproducibility (CV = $4.1 \%$ between replicates), indicating that it is sensitive to detect even low concentrations of IFN- $\alpha_{2 b}$. We found a significant correlation between the concentrations of IFN $-\alpha_{2 b}$ in plasma and sSIGLEC- $1\left(r^{2}=0.27, P=5.1 \times\right.$ 

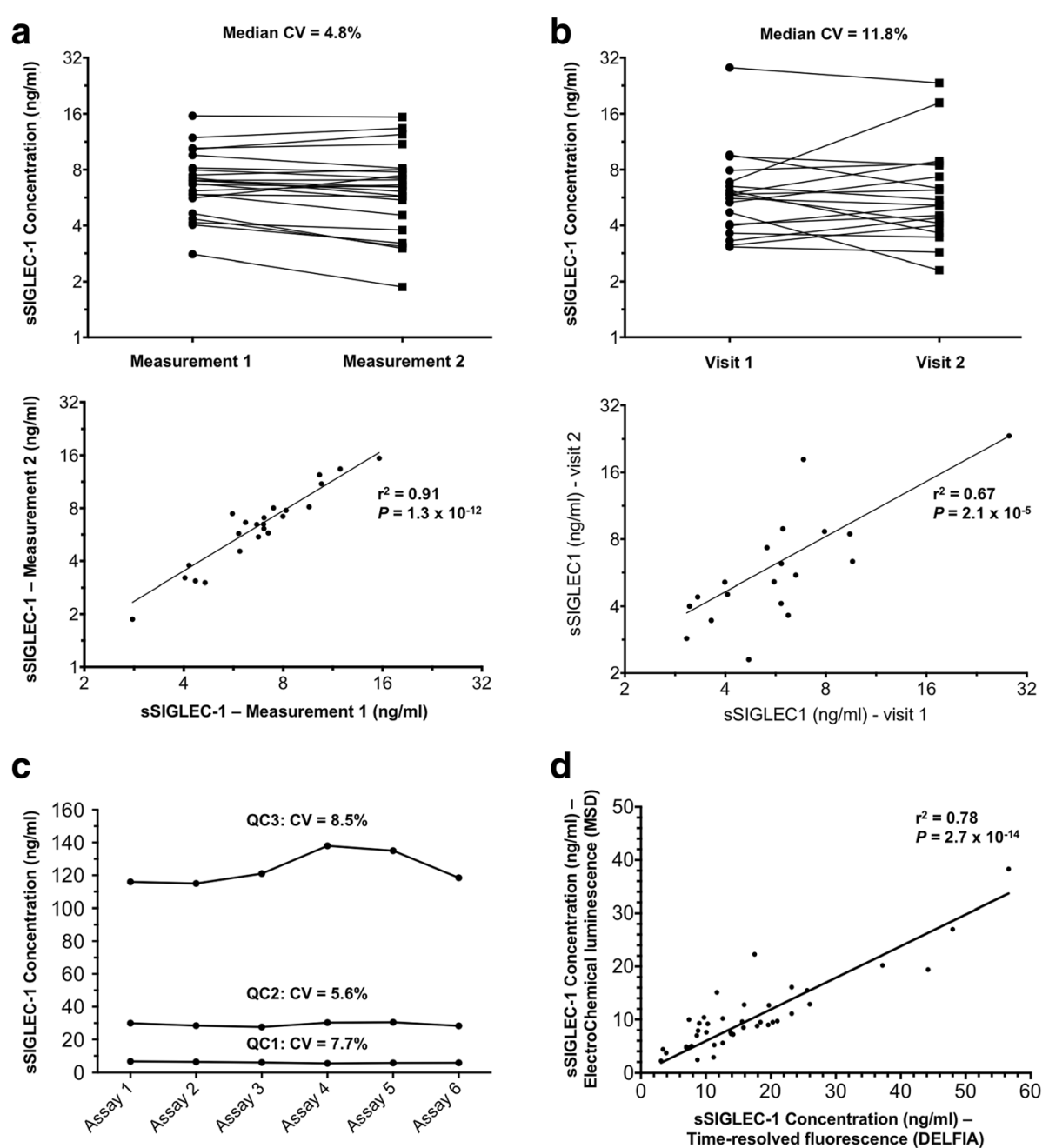

Fig. 1 sSIGLEC-1 stability and assay performance. a Data depict the inter-assay (technical) variation of the time-resolved fluorescence (TRF) sSIGLEC-1 immunoassay. Data were obtained from the measurement of the same plasma sample from 23 healthy donors on two independent assays, performed 308 days apart. b Data depict the intra-individual (biological) variation of sSIGLEC-1 concentration between two visits of the same donor. Longitudinal variation was assessed in 19 independent healthy donors using plasma samples collected at each separate visit. Median time between visits was 378 days (minimum $=239$ days, maximum $=519$ days). $\mathbf{c}$ Technical variation of the electrochemical luminescence $(E C L)$ sSIGLEC-1 immunoassay using the Meso Scale Discovery (MSD) platform. Assay stability was assessed in three reference quality control (QC) pools of serum samples with increasing SSIGLEC-1 concentrations (QC1 $=6.0 \mathrm{ng} / \mathrm{ml}, \mathrm{QC2}=29.2 \mathrm{ng} / \mathrm{ml}$ and QC3 $=123.9 \mathrm{ng} / \mathrm{ml})$, measured in each assay over a 2-day period. $\mathbf{d}$ Correlation between TRF (DELFIA) and ECL (MSD)-based immunoassays. Measurements were performed in independent serum aliquots from a subset of 41 SLE patients from cohort 3. $P$ values were calculated by linear regression. CV coefficient of variation, DELFIA dissociation-enhanced lanthanide fluorescent immunoassay, SSIGLEC-1 soluble sialic acid binding Ig-like lectin 1

$10^{-4}$; Fig. 3c) as well as the IFN signature $\left(r^{2}=0.34, P=\right.$ $5.6 \times 10^{-5}$; see Additional file 2), although both were less pronounced than the observed correlation between sSIGLEC- 1 and the IFN transcriptional signature.

In our sample of 34 SLE patients from the discovery cohort, sSIGLEC-1 concentrations and the SLE Disease Activity Index (SLEDAI) were not correlated $\left(r^{2}=0.10\right.$, $P=0.07$; Fig. $3 \mathrm{~d}$ ). This result is consistent with previous evidence showing a lack of association of other common serological disease markers, including various intra-nuclear autoantibodies, elevated B-cell activating factor of the tumour necrosis factor family (BAFF) levels and hypocomplementaemia, as well as the whole blood IFN signature, with disease activity scores such as the SLEDAI [6].

\section{Increased sSIGLEC-1 concentration is associated with renal involvement}

Having assessed that sSIGLEC-1 is an IFN-regulated marker that can be detected in the circulation, we next investigated its potential as a clinical biomarker in SLE. Similarly to surface SIGLEC-1 expression, sSIGLEC-1 concentrations were markedly increased in SLE patients (10.4 $\mathrm{ng} / \mathrm{ml}, 95 \%$ CI $8.8-12.2)$ compared to healthy 

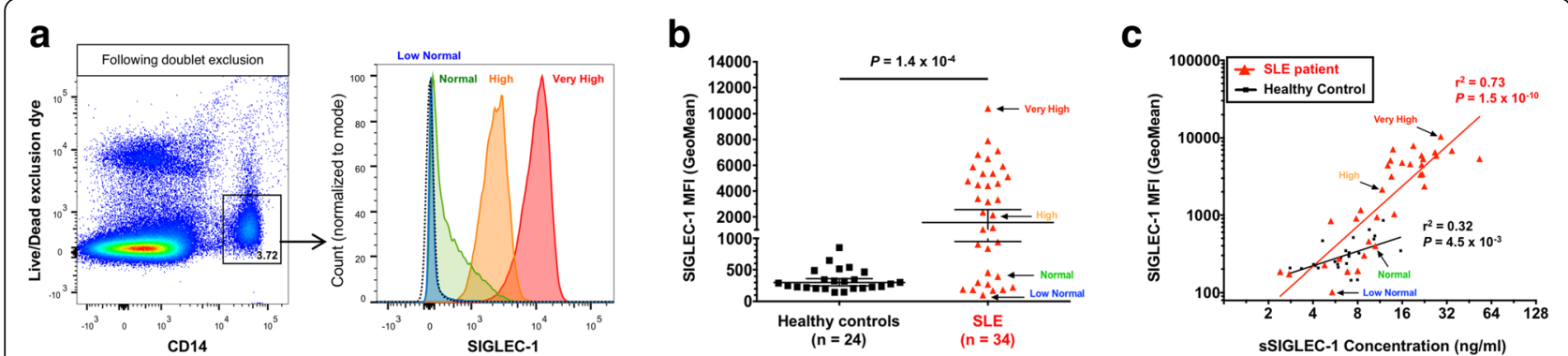

Fig. 2 Soluble SIGLEC-1 is a surrogate for the surface expression of SIGLEC-1 on CD14 ${ }^{+}$monocytes. a Gating strategy for the delineation of CD14 $4^{+}$monocytes, following single-cell discrimination. Histograms depict the distribution of SIGLEC-1 expression on the surface of CD14 ${ }^{+}$ monocytes obtained by flow cytometry in illustrative donors expressing low normal (blue), normal (green), high (orange) or very high (red) levels of SIGLEC-1. Dotted line represents the background expression of SIGLEC-1 in live lymphocytes, which are known not to express SIGLEC-1. $\mathbf{b}$ Scatter plot depicts the frequency (geometric mean $\pm 95 \% \mathrm{CI}$ ) of SIGLEC-1 expression on the surface of CD $14^{+}$monocytes in a discovery cohort (cohort 1) of healthy donors ( $N=24$; black squares) and SLE patients ( $N=34$; red triangles). $P$ value was calculated using a two-tailed nonparametric Mann-Whitney test. c Correlation between SIGLEC-1 mean fluorescence intensity (MFI) on CD14 $4^{+}$monocytes obtained by flow cytometry and the corresponding SSIGLEC-1 concentration in the healthy control and SLE patient groups. $P$ value was calculated by linear regression. Illustrative SIGLEC-1 low normal, normal, high and very high SLE patients shown in (a) are highlighted in (b) and (c). SIGLEC-1 sialic acid binding Ig-like lectin 1, sSIGLEC-1 soluble SIGLEC-1, SLE systemic lupus erythematosus

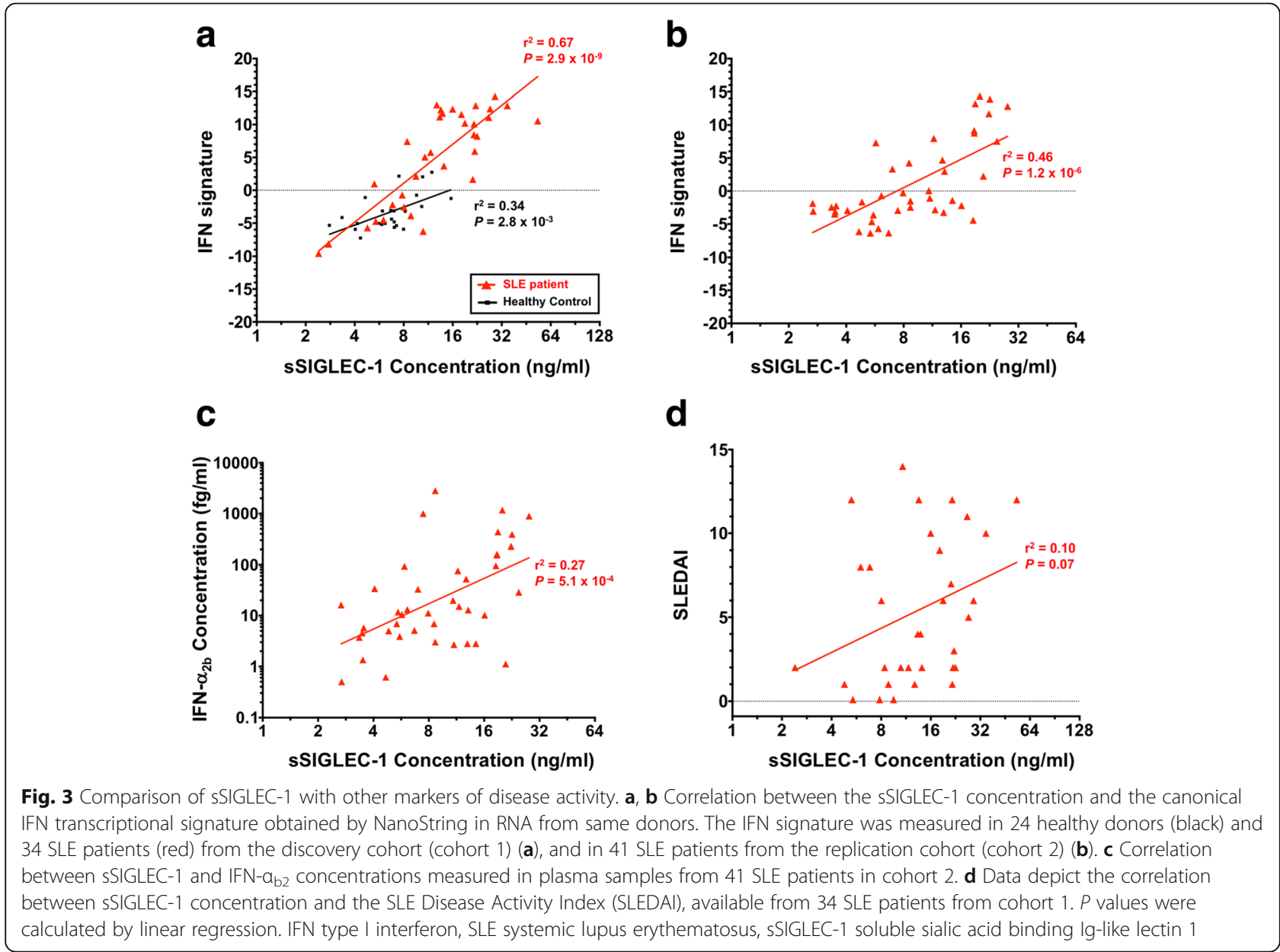


controls $\left(5.78 \mathrm{ng} / \mathrm{ml}, 95 \%\right.$ CI 5.5-6.0, $P=9.6 \times 10^{-12}$; Fig. 4a) in the combined discovery and replication cohorts of 75 SLE patients and 504 healthy donors. In addition to SLE, we also assessed sSIGLEC-1 concentrations in a cohort of SSc patients $(n=50$ presenting with limited cutaneous and 49 with diffuse cutaneous SSc), another systemic autoimmune disease where type I IFN and overt monocyte/macrophage activation have been suggested to play an important aetiological role [18, 24]. Consistent with previous data showing an increased expression of SIGLEC-1 on the surface of $\mathrm{CD} 14^{+}$monocytes in SSc patients [18], we found evidence for an increased concentration of sSIGLEC-1 in serum samples from SSc (8.49 $\mathrm{ng} / \mathrm{ml}, 95 \%$ CI $8.5-10.5)$ compared to matched healthy controls $\left(7.07,95 \% \mathrm{CI} 6.7-8.6, P=8.3 \times 10^{-3}\right.$; Fig. $4 \mathrm{~b})$. The distribution of sSIGLEC-1 in SSc was similar to the SLE patients and the increased concentrations were maintained in both patients with limited cutaneous SSc or diffuse SSc $(P=0.02$ and $P=0.05$, respectively; Fig. $4 b)$.

To assess the potential clinical application of sSIGLEC-1, we measured sSIGLEC-1 levels in serum from 656 SLE patients with available clinical information (cohort 3). We observed that the concentrations of sSIGLEC-1 were

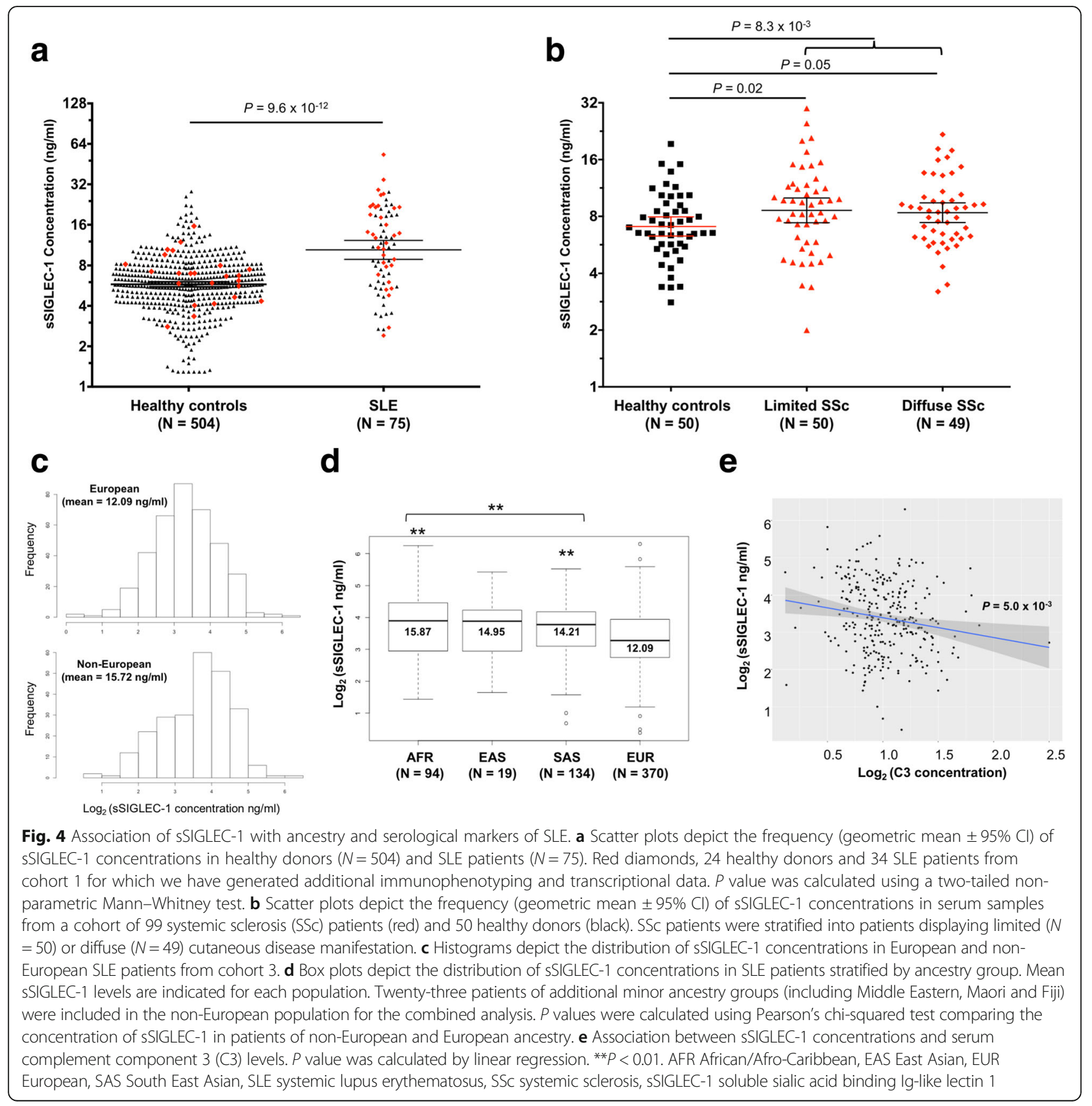


significantly higher in non-European patients $(15.7 \mathrm{ng} / \mathrm{ml}$, 95\% CI 13.52-17.92) compared to those of European ancestry $\left(12.1 \mathrm{ng} / \mathrm{ml}, 95 \%\right.$ CI $11.25-12.94, P=2.7 \times 10^{-3}$; Fig. 4c). Soluble SIGLEC-1 levels were similarly elevated among the different non-European populations (Fig. 4d), and were therefore combined into a single group to increase statistical power. Increased disease severity-and particularly incidence of renal disease-has been documented in patients of non-European ancestry [25-27]. In agreement with this observation, in our study, non-European SLE patients presented with an increased history of renal complications (Table 1), thus suggesting that the increased SSIGLEC-1 concentrations reflected the increased disease severity in non-European patients.

In addition to the ancestry-related changes, we found that sSIGLEC-1 levels were associated with lower levels of serum complement component 3 (C3) $\left(P=5.0 \times 10^{-3}\right.$; Fig. 4e), but not with other common serological markers of SLE such as C-reactive protein or anti-nuclear autoantibody levels (see Additional file 3). This association was observed in both European and non-European patients and remained present even when adjusting for the presence of nephritis. Furthermore, we found that increased sSIGLEC-1 concentrations were associated with a history of renal disease in the combined patient population $(P=0.01$; Table 2). Although the number of patients with active nephritis was limited in our study, we found that sSIGLEC-1 concentrations were strongly associated with duration of renal disease, with significantly higher levels observed in patients with active renal disease, and gradually declining with time (Fig. 5a), likely reflecting improved disease management since the last episode of active nephritis. The risk of renal complications/nephritis in patients with very high concentrations of sSIGLEC-1 was much more pronounced in European patients $(\mathrm{OR}=$ 1.65) compared to non-European patients $(\mathrm{OR}=1.16$; Table 2), and was maintained after adjusting for the association of sSIGLEC-1 with low C3 and C4 levels, which are predictors for renal disease $(\mathrm{OR}=1.96,95 \% \mathrm{CI}$ $1.10-3.45 ; P=0.021)$. Since sSIGLEC-1 could be physiologically excreted through the kidney, we also investigated whether increased sSIGLEC-1 concentrations could be associated with decreased kidney function in patients with renal disease. We found no evidence for an association between kidney function, as measured by the patients' estimated glomerular filtration rate (eGFR), and sSIGLEC-1 concentration (Fig. 5b). The association of sSIGLEC-1 and renal nephritis was maintained after adjusting for the effect of eGFR (OR $=1.49,95 \%$ CI 1.02-2.17; $P=0.04)$. These data suggest that decreased renal function in patients with renal nephritis does not fully explain the association with increased sSIGLEC-1 concentrations observed in this study.

In addition to the association of SSIGLEC-1 with renal complications, we also observed a similar trend towards increased risk of haematological complications in patients with high concentrations of sSIGLEC-1, although not reaching statistical significance in our analysis $(\mathrm{OR}=1.35$, $P=0.09$; Table 2). Further supporting the potential use of sSIGLEC-1 as a biomarker of disease severity, a higher frequency of patients with high levels of sSIGLEC-1 had a history of treatment with biologics $(\mathrm{OR}=1.20, P=0.04$ in the combined population; Table 2), usually associated with patients with poor disease management who have not responded to standard treatment options. This remained the case even when we adjusted for history of renal complication as a confounding factor for biologics use.

\section{Discussion}

Recent advances in medical research have led to the development of a breadth of novel treatment options. The characterisation of biomarkers that identify the exact pathophysiological mechanism underpinning the clinical manifestations in each patient has thus become a

Table 2 Association of sSIGLEC-1 with clinical parameters of SLE

\begin{tabular}{|c|c|c|c|c|c|c|c|c|c|}
\hline \multirow[t]{2}{*}{ Clinical parameter } & \multicolumn{3}{|c|}{ Combined } & \multicolumn{3}{|l|}{ EUR } & \multicolumn{3}{|l|}{ Non-EUR } \\
\hline & $\overline{N \text { (total) }}$ & $\mathrm{OR}^{\mathrm{a}}(95 \% \mathrm{Cl})$ & $P$ value $^{b}$ & $N$ (total) & $\mathrm{OR}^{\mathrm{a}}(95 \% \mathrm{Cl})$ & $P$ value $^{\mathrm{b}}$ & $N$ (total) & $\mathrm{OR}^{\mathrm{a}}(95 \% \mathrm{Cl})$ & $P$ value ${ }^{b}$ \\
\hline Renal (nephritis) & 156 & $1.53(1.10-2.15)$ & 0.01 & 75 & $1.65(1.09-2.52)$ & 0.02 & 81 & $1.16(0.60-2.25)$ & 0.67 \\
\hline Haematological & 291 & $1.35(0.95-1.92)$ & 0.09 & 185 & $1.34(0.80-2.20)$ & 0.25 & 106 & $1.28(0.72-2.25)$ & 0.4 \\
\hline Biologics ${ }^{c}$ & 35 & $1.20(1.01-1.42)$ & 0.04 & 23 & $1.18(0.90-1.18)$ & 0.19 & 12 & $0.95(0.70-1.23)$ & 0.71 \\
\hline Neurological & 78 & $1.01(0.76-1.34)$ & 0.95 & 36 & $0.87(0.63-1.18)$ & 0.37 & 42 & $0.83(0.39-1.76)$ & 0.64 \\
\hline Admission & 226 & $1.18(0.81-1.71)$ & 0.39 & 140 & $145(0.90-2.37)$ & 0.13 & 86 & $0.60(0.30-1.21)$ & 0.16 \\
\hline Corticosteroid use & 302 & $0.98(0.69-1.41)$ & 0.96 & 174 & $0.96(0.57-1.57)$ & 0.86 & 128 & $0.88(0.49-1.56)$ & 0.35 \\
\hline
\end{tabular}

Association of sSIGLEC-1 concentration with clinical parameters recorded from SLE patients occurring since their disease diagnosis and up to the time of their visit CI confidence interval, EUR patients of European ancestry, non-EUR patients of non-European ancestry, SLE systemic lupus erythematosus, sSIGLEC-1 soluble sialic acid binding Ig-like lectin 1

${ }^{a}$ Odds ratio (OR) calculated on the group of patients with sSIGLEC levels $>95$ th percentile

${ }^{\mathrm{b}} \mathrm{P}$ values were calculated in each group using a logistical regression model, where the SLE patients were divided into groups based on sSIGLEC1 serum level centiles ( $<50$ th centile, 51st-74th centile, 75th-95th centile and $>95$ th centile)

${ }^{\mathrm{c}}$ Patients treated with biologic drugs at the time of visit 

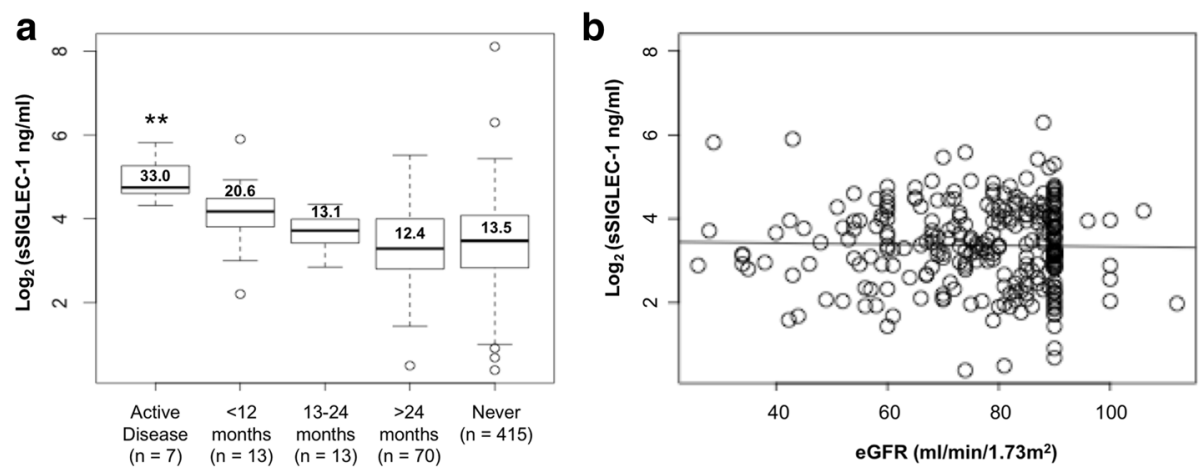

Fig. 5 SSIGLEC-1 concentrations are increased in patients with active renal disease. a Box plots depict the distribution of sSIGLEC-1 concentrations in SLE patients with renal complications/nephritis, stratified by time since last episode of active nephritis. Information on duration of renal disease was available for 103 of the 156 patients with reported renal complications/nephritis in cohort 3. Mean sSIGLEC-1 levels are indicated for each population. $P$ values were calculated using two-tailed Student's $t$ tests comparing SSIGLEC-1 concentrations between patients in each disease duration group and patients who never developed renal complications. $\mathbf{b}$ Correlation between SSIGLEC-1 levels and renal function, measured by estimated glomerular filtration rate (eGFR) in 280 SLE patients from cohort 3. ${ }^{*} P<0.01$. eGFR estimated glomerular filtration rate, SSIGLEC-1 soluble sialic acid binding lg-like lectin 1

priority to allow the advent of a truly personalised medicine approach to human complex diseases. In systemic autoimmune and inflammatory diseases, chronic IFN signalling has been shown to be directly involved in the pathogenesis of the diseases, most notably in SLE [28]. This observation has led to the development of therapeutic strategies to target IFN- $\alpha$, which are currently being tested in the clinic $[29,30]$. There is therefore an urgent need to develop robust and sensitive biomarkers to identify patients with an active IFN response who would be more likely to benefit from anti-IFN- $\alpha$ therapy.

In the present study, we show that a circulating form of the surface-bound SIGLEC-1 receptor can be detected in human plasma/serum, and developed a sensitive immunoassay to measure the circulating concentrations of sSIGLEC-1. To our knowledge we are the first to detect the presence of a soluble SIGLEC-1 isoform in humans. Our current data do not allow us to determine the origin of sSIGLEC-1, and further work is needed to assess whether the soluble isoform is generated through alternative splicing or by proteolytic shedding of the membrane-bound receptor. A key feature of this bioassay is the limited sample requirements, making it amenable-as compared to a flow cytometric assay of surface SIGLEC-1to screen large numbers of samples. We therefore propose that quantification of sSIGLEC-1 using our immunoassay is an alternative to flow cytometric endpoints, or the classical IFN signature or its PCR-analysed surrogate [6], and will result in a more robust, simpler and less expensive measure of SIGLEC-1 expression and the IFN signature.

Other plasma/serum IFN-regulated biomarkers have been described in the literature, most notably IFN- $\alpha$ and IFN- $\gamma$-inducible protein 10 (IP-10) [12]. However, the protein stability, cell-type specificity and much higher concentrations of sSIGLEC-1 are major practical advantages of our immunoassay. Moreover, measuring all 16 different IFN- $\alpha$ subtypes currently requires access to naturally occurring high-affinity anti-IFN- $\alpha$ antibodies that are not readily available [23], which in combination with the much lower sample requirement compared to the SIMOA assay makes the sSIGLEC-1 assay ideally suited for high-throughput screening, including the retrospective testing of samples collected from completed clinical studies or cohorts for flares of IFN signalling and/or response to treatment of large cohorts and retrospective studies. Recently, a study has reported that plasma concentrations of presepsin (PSEP), a product of $\mathrm{CD}^{+} 4^{+}$monocyte cleavage, is increased in patients with SLE and other autoimmune and inflammatory diseases [31]. Although the study was limited to a small sample size and available clinical data, the wide spread of PSEP levels measured in SLE patients and the suggested association with disease activity are consistent with our data and support a role for activated monocyte/ macrophages in the aetiology of SLE and other related autoimmune diseases. Further work will be required to investigate the relation between sSIGLEC-1 and PSEP, and the putative diagnostic and/or prognostic value of using a combination of biomarkers associated with exacerbated monocyte activation for the clinical stratification of patients with a systemic activation of the innate immune system.

Clinically, we identified marked ancestry-related differences in sSIGLEC-1 levels among SLE patients, which were consistent with the higher disease severity in patients of non-European ancestry. In agreement with this hypothesis, sSIGLEC-1 levels were also associated with lower levels of $\mathrm{C} 3$, a classical serologic marker of the disease. Furthermore, our data provide evidence that high sSIGLEC-1 levels could be predictive of active renal and haematological complications, particularly in patients of European ancestry. 
These findings clearly underscore the importance of large, well-characterised, clinical cohorts to estimate the confounding effects of ancestry. In this study we had access to very limited numbers of non-European healthy controls, which prevented us from investigating whether steady-state sSIGLEC-1 levels could also be altered in populations of non-European ancestry. However, the association of sSIGLEC-1 concentrations with overall increased disease severity was consistently maintained in both groups of patients. A possible explanation for the more modest predictive capacity observed in non-European patients is an increased disease heterogeneity, which could reflect a reduced dependency on the type I IFN pathway for disease severity and late-stage organ damage in this group of patients. A limitation of this study was the number of available patients with active disease manifestationsnamely, nephritis - which reduced the power to investigate the predictive capacity of high sSIGLEC-1 levels for the identification of patients with active disease. Further work will now be required to validate these findings in additional autoimmune and inflammatory diseases associated with a chronic activation of the innate immune system using large and clinically well-characterised patient populations, as well as to extend the findings to non-European cohorts.

There has been considerable interest in developing drugs targeting the IFN- $\alpha$ signalling pathway to treat conditions associated with chronic IFN signalling. Our assay could also have utility in clinical trials; for example, for the selection of patients who could benefit the most from such inhibition of IFN- $\alpha$. Conversely, IFN- $\alpha$ is also one of the most used compounds in cytokine therapy. However, its immunomodulatory properties may result in various autoimmune manifestations, with reported incidence of $4-19 \%$ in patients undergoing IFN- $\alpha$ therapy [32]. We therefore hypothesise that these adverse events may be avoided if the background IFN signature is known and therapy is adjusted to avoid the excessive IFN signalling known to be a factor in the promotion of secondary autoimmunity in these patients. Moreover, it has been recently suggested that the detection of an IFN signature in peripheral blood is associated with poor response to both B-cell depleting therapy (rituximab) and anti-IL-6R treatment (tocilizumab) in rheumatoid arthritis patients $[33,34]$. These data suggest that sSIGLEC-1 could be useful in prediction to therapeutic responses, and supports a broader application of this assay in the context of patient stratification for clinical trials.

\section{Conclusions}

In this study we report the development of a sensitive immunoassay to detect circulating concentrations of sSIGLEC-1 in plasma/serum. Taken together, our findings suggest that sSIGLEC-1 is a marker of monocyte and macrophage activation, which is critically implicated in the progression of several autoimmune and inflammatory diseases, such as SLE and SSc. In combination with additional available IFN-regulated biomarkers, the sSIGLEC-1 bioassay could help improve our capacity to dissect the molecular and clinical heterogeneity of complex conditions associated with an overt IFN response, and identify subsets of common and rare autoimmune and inflammatory diseases, collectively classified as interferonopathies. We have also shown that increased sSIGLEC-1 concentrations could, with further studies, have a clinical application in predicting increased risk of developing renal complications, one of the most severe clinical complications of SLE.

\section{Additional files}

Additional file 1: Table S1. Custom NanoString probe sequences used to measure expression of the 56 IFN-regulated genes defining the transcriptional IFN signature in this study and eight housekeeping genes used to normalise expression levels between donors. (XLSX 13 kb)

Additional file 2: Figure S1. Concentration of $I F N-a_{b_{2}}$ is associated with the IFN transcriptional signature. Data depict correlation between plasma IFN- $a_{b_{2}}$ concentration and transcriptional IFN signature in 41 SLE patients from cohort 2. (PDF $35 \mathrm{~kb}$ )

Additional file 3: Figure S2. Association of sSIGLEC-1 with serological markers of SLE. $\mathbf{a}, \mathbf{b}$ Data depict the association of SSIGLEC-1 concentrations with C-reactive protein (CRP) levels (a) and with disease-specific anti-nuclear autoantibody (ANA) titres (b). $P$ values were calculated by linear regression. (PDF $347 \mathrm{~kb}$ )

\begin{abstract}
Abbreviations
ACR: American College of Rheumatology; C3: Serum complement component 3; Cl: Confidence interval; CV: Coefficient of variation; DELFIA: Dissociation-enhanced lanthanide fluorescent immunoassay; ECL: Electrochemical luminescence; ELISA: Enzyme-linked immunosorbent assay; IFN: Type I interferon; IP-10: IFN- $\gamma$-inducible protein 10; IRG: Type I interferon regulated genes; OR: Odds ratio; PBMC: Peripheral blood mononuclear cell; SIGLEC-1: Sialic acid binding Ig-like lectin 1; SLE: Systemic lupus erythematosus; SLEDAI: SLE Disease Activity Index; SSc: Systemic sclerosis; sSIGLEC-1: Soluble SIGLEC-1; TRF: Time-resolved fluorescence
\end{abstract}

\section{Acknowledgements}

The authors gratefully acknowledge the participation of all NIHR Cambridge BioResource volunteers, and thank the NIHR Cambridge BioResource centre and staff for their contribution. They thank the National Institute for Health Research and NHS Blood and Transplant. The authors thank Neil Walker and Helen Schuilenburg from the Cambridge Institute for Medical Research,

University of Cambridge for data management. They thank members of the Cambridge BioResource SAB and management committee for their support and the National Institute for Health Research Cambridge Biomedical Research Centre for funding. The authors also thank Helen Stevens, Gill Coleman, Sarah Dawson, Simon Duley, Meeta Maisuria and Sumiyya Mahmood from the Cambridge Institute for Medical Research, University of Cambridge for preparation of DNA and PBMC samples. They also thank Emma Jones from AstraZeneca for the use of the NanoString instrument and Claudia Gonzalez-Lopez from the Weatherall Institute of Molecular Medicine, Oxford, for the use of the Simoa HD-1 analyser.

Funding

This work was funded by the JDRF (9-2011-253), the Wellcome (WT061858/ 091157) and the National Institute for Health Research Cambridge Biomedical Research Centre. This research was also funded/supported by the National Institute for Health Research Biomedical Research Centre based at Guy's and St Thomas' NHS Foundation Trust and King's College London. RCF 
is funded by a JDRF Advanced post-doctoral fellowship (2-APF-2017-420-A$\mathrm{N})$.

\section{Availability of data and materials}

The datasets generated during the present study are available from the corresponding author on reasonable request.

\section{Authors' contributions}

JAT and RCF conceived the study. TJV, LSW, JAT and RCF designed the experiments. JJO, DBR, CLP, PC, OA-A, KB and RCF performed the experiments. JJO, SK, ARG, KB and RCF analysed the data and interpreted the results. SK, SM RS and TJV provided patient samples. RCF wrote the manuscript with input from all co-authors. All authors read and approved the final manuscript.

\section{Ethics approval and consent to participate}

All samples and information were collected with written and signed informed consent after approval from the relevant research ethics committees (REC numbers 05/Q0106/20, 07/H0718/49, 08/H0308/153; and NRES Committee London-Hampstead, HRA, REC number 6398).

\section{Consent for publication}

Not applicable.

\section{Competing interests}

$J \mathrm{JO}$ is an employee of GlaxoSmithKline pharmaceuticals. The other authors declare that they have no competing interests.

\section{Publisher's Note}

Springer Nature remains neutral with regard to jurisdictional claims in published maps and institutional affiliations.

\section{Author details}

'Department of Medical Genetics, JDRF/Wellcome Diabetes and Inflammation Laboratory, NIHR Cambridge Biomedical Research Centre, Cambridge Institute for Medical Research, University of Cambridge, Cambridge, UK. ${ }^{2}$ Division of Genetics and Molecular Medicine and Division of Immunology, Infection and Inflammatory Disease, King's College London, Great Maze Pond, London, UK. J.JDRF/Wellcome Diabetes and Inflammation Laboratory, Wellcome Centre for Human Genetics, Nuffield Department of Medicine, NIHR Oxford Biomedical Research Centre, University of Oxford, Roosevelt Drive, Oxford, UK. ${ }^{4}$ NIHR Cambridge Biomedical Research Centre, Core Biochemical Assay Laboratory, Cambridge, UK. ${ }^{5}$ UCL Centre for Rheumatology and Connective Tissue Diseases, UCL Medical School, Royal Free Hospital Campus, Rowland Hill Street, London, UK.

Received: 16 February 2018 Accepted: 20 June 2018

Published online: 27 July 2018

\section{References}

1. Preble OT, Black RJ, Friedman RM, Klippel JH, Vilcek J. Systemic lupus erythematosus: presence in human serum of an unusual acid-labile leukocyte interferon. Science (80). 1982;216:429-31.

2. Obermoser G, Pascual V. The interferon-a signature of systemic lupus erythematosus. Lupus. 2010;19:1012-9.

3. Morimoto AM, Flesher DT, Yang J, Wolslegel K, Wang X, Brady A, et al. Association of endogenous anti-interferon-a autoantibodies with decreased interferon-pathway and disease activity in patients with systemic lupus erythematosus. Arthritis Rheum. 2011;63:2407-15.

4. Baechler EC, Batliwalla FM, Karypis G, Gaffney PM, Ortmann WA, Espe KJ, et al. Interferon-inducible gene expression signature in peripheral blood cells of patients with severe lupus. Proc Natl Acad Sci U S A. 2003;100:2610-5.

5. Bennett L, Palucka AK, Arce E, Cantrell V, Borvak J, Banchereau J, et al. Interferon and granulopoiesis signatures in systemic lupus erythematosus blood. J Exp Med. 2003;197:711-23.

6. Kennedy WP, Maciuca R, Wolslegel K, Tew W, Abbas AR, Chaivorapol C, et al. Association of the interferon signature metric with serological disease manifestations but not global activity scores in multiple cohorts of patients with SLE. Lupus Sci Med. 2015;2:e000080.

7. Banchereau R, Hong S, Cantarel B, Baldwin N, Baisch J, Edens M, et al. Personalized Immunomonitoring uncovers molecular networks that stratify lupus patients. Cell. 2016;165:551-65.
8. Strauß R, Rose T, Flint SM, Klotsche J, Häupl T, Peck-Radosavljevic M, et al. Type I interferon as a biomarker in autoimmunity and viral infection: a leukocyte subset-specific analysis unveils hidden diagnostic options. J Mol Med. 2017:95:753-65.

9. Landolt-Marticorena C, Bonventi G, Lubovich A, Ferguson C, Unnithan T, Su $J$, et al. Lack of association between the interferon-a signature and longitudinal changes in disease activity in systemic lupus erythematosus. Ann Rheum Dis. 2009;68:1440-6.

10. Petri M, Singh S, Tesfasyone H, Dedrick R, Fry K, Lal PG, et al. Longitudinal expression of type I interferon responsive genes in systemic lupus erythematosus. Lupus. 2009;18:980-9.

11. Biesen R, Demir C, Barkhudarova F, Grün JR, Steinbrich-Zöllner M, Backhaus M, et al. Sialic acid-binding lg-like lectin 1 expression in inflammatory and resident monocytes is a potential biomarker for monitoring disease activity and success of therapy in systemic lupus erythematosus. Arthritis Rheum. 2008:58:1136-45.

12. Rose T, Grützkau A, Hirseland H, Huscher D, Dähnrich C, Dzionek A, et al. IFNa and its response proteins, IP-10 and SIGLEC-1, are biomarkers of disease activity in systemic lupus erythematosus. Ann Rheum Dis. 2013;72:1639-45.

13. Rose T, Grützkau A, Klotsche J, Enghard P, Flechsig A, Keller J, et al. Are interferon-related biomarkers advantageous for monitoring disease activity in systemic lupus erythematosus? A longitudinal benchmark study. Rheumatology. 2017;56:1618-26.

14. O'Neill ASG, van den Berg TK, Mullen GED. Sialoadhesin-a macrophage-restricted marker of immunoregulation and inflammation. Immunology. 2013;138:198-207.

15. Hartnell A, Steel J, Turley $H$, Jones $M$, Jackson DG, Crocker PR. Characterization of human sialoadhesin, a sialic acid binding receptor expressed by resident and inflammatory macrophage populations. Blood. 2001;97:288-96.

16. Izquierdo-Useros N, Lorizate M, Puertas MC, Rodriguez-Plata MT, Zangger N,

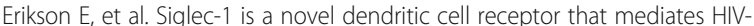
1 trans-infection through recognition of viral membrane gangliosides. PLoS Biol. 2012;10:e1001448.

17. Xiong Y-S, Cheng Y, Lin Q-S, Wu A-L, Yu J, Li C, et al. Increased expression of Siglec-1 on peripheral blood monocytes and its role in mononuclear cell reactivity to autoantigen in rheumatoid arthritis. Rheumatol. 2014;53:250-9.

18. York MR, Nagai T, Mangini AJ, Lemaire R, van Seventer JM, Lafyatis R. A macrophage marker, Siglec-1, is increased on circulating monocytes in patients with systemic sclerosis and induced by type I interferons and tolllike receptor agonists. Arthritis Rheum. 2007;56:1010-20.

19. Bao G, Han Z, Yan Z, Wang Q, Zhou Y, Yao D, et al. Increased Siglec-1 expression in monocytes of patients with primary biliary cirrhosis. Immunol Investig. 2010:39:645-60.

20. Lisney AR, Szelinski F, Reiter K, Burmester GR, Rose T, Dörner T. High maternal expression of SIGLEC1 on monocytes as a surrogate marker of a type I interferon signature is a risk factor for the development of autoimmune congenital heart block. Ann Rheum Dis. 2017;76:1476-80.

21. van den Hoogen F, Khanna D, Fransen J, Johnson SR, Baron M, Tyndall A, et al. 2013 classification criteria for systemic sclerosis: an American College of Rheumatology/European League Against Rheumatism collaborative initiative. Ann Rheum Dis. 2013;72:1747-55.

22. Ferreira RC, Guo H, Coulson RMR, Smyth DJ, Pekalski ML, Burren OS, et al. A type I interferon transcriptional signature precedes autoimmunity in children genetically at risk for type 1 diabetes. Diabetes. 2014:63:2538-50.

23. Rodero MP, Decalf J, Bondet V, Hunt D, Rice GI, Werneke S, et al. Detection of interferon alpha protein reveals differential levels and cellular sources in disease. J Exp Med. 2017;214:1547-55.

24. Wu M, Assassi S. The role of type 1 interferon in systemic sclerosis. Front Immunol. 2013:4:266.

25. Petri M, Perez-Gutthann S, Longenecker JC, Hochberg M. Morbidity of systemic lupus erythematosus: role of race and socioeconomic status. Am J Med. 1991:91:345-53.

26. Alarcón GS, McGwin G Jr, Petri M, Ramsey-Goldman R, Fessler BJ, Vilá LM, et al. Time to renal disease and end-stage renal disease in PROFILE: a multiethnic lupus cohort. PLoS Med. 2006;3:e396.

27. Bruce IN, O'Keeffe AG, Farewell V, Hanly JG, Manzi S, Su L, et al. Factors associated with damage accrual in patients with systemic lupus erythematosus: results from the Systemic Lupus International Collaborating Clinics (SLICC) Inception Cohort. Ann Rheum Dis. 2015:74:1706-13.

28. Hooks JJ, Moutsopoulos HM, Geis SA, Stahl NI, Decker JL, Notkins AL. Immune interferon in the circulation of patients with autoimmune disease. N Engl J Med. 1979;301:5-8. 
29. Lauwerys BR, Ducreux J, Houssiau FA. Type I interferon blockade in systemic lupus erythematosus: where do we stand? Rheumatol. 2014;53:1369-76.

30. Oon S, Wilson NJ, Wicks I. Targeted therapeutics in SLE: emerging strategies to modulate the interferon pathway. Clin Trans Immunol. 2016;5:e79.

31. Tanimura S, Fujieda Y, Kono M, Shibata Y, Hisada R, Sugawara E, et al. Clinical significance of plasma presepsin levels in patients with systemic lupus erythematosus. Mod Rheumatol. 2017:1-7. https://doi.org/10.1080/ 14397595.2017.1408755.

32. Ioannou Y, Isenberg DA. Current evidence for the induction of autoimmune rheumatic manifestations by cytokine therapy. Arthritis Rheum. 2000;43: $1431-42$.

33. Raterman HG, Vosslamber $S$, de Ridder $S$, Nurmohamed MT, Lems WF, Boers $\mathrm{M}$, et al. The interferon type I signature towards prediction of non-response to rituximab in rheumatoid arthritis patients. Arthritis Res Ther. 2012;14:1-10.

34. Sanayama Y, Ikeda K, Saito Y, Kagami S, Yamagata M, Furuta S, et al. Prediction of therapeutic responses to tocilizumab in patients with rheumatoid arthritis: biomarkers identified by analysis of gene expression in peripheral blood mononuclear cells using genome-wide DNA microarray. Arthritis Rheumatol. 2014;66:1421-31.

Ready to submit your research? Choose BMC and benefit from:

- fast, convenient online submission

- thorough peer review by experienced researchers in your field

- rapid publication on acceptance

- support for research data, including large and complex data types

- gold Open Access which fosters wider collaboration and increased citations

- maximum visibility for your research: over $100 \mathrm{M}$ website views per year

At BMC, research is always in progress.

Learn more biomedcentral.com/submissions 